\title{
La poesía medieval como objeto de estudio en el siglo XXI: evanescencia y fijación en el medio digital
}

\author{
Medieval Poetry as object of study in the $21^{\text {st }}$ c.: \\ Evanescence and Fixation in the Digital Medium
}

\author{
Gimena DEL RIO RIANDE, \\ Clara I. MARTÍNEZ CANTÓN y Elena GONZÁLEZ-BLANCO \\ (IIBICRIT, CONICET / UNED / UNED)
}

gdelrio@conicet.gov.ar / cimartinez@flog.uned.es / egonzalezblanco@flog.uned.es ORCID ID: 0000-0002-8997-5415 / 0000-0003-0781-2418 / 0000-0002-0448-1812

\begin{abstract}
Digital humanities are considered RESUMEN: Las humanidades digitales, como una today as an ideal tool to approach Medieval poetry forma de aproximación a la poesía medieval, son un that has been transmitted through different means: tema que llama la atención en la actualidad, sobre orally, in print and through the digital medium. todo considerando que la poesía medieval ha pasado Medieval poetry has consequently occupied a por difusión oral, impresa y digital. La poesía privileged position in academic studies, with new medieval en el medio digital ha ocupado un lugar texts to examine such as catalogues, indexes, privilegiado en los estudios académicos, de donde repositories, databases, etcetera. Also, digitalize- surgen nuevos elementos a estudiar, como son: tion enables the use of other tools which are hybrid catálogos, índices, repositorios, bases de datos, etc. databases such as the POSDATA project. Además, la digitalización posibilita el uso de otras herramientas que constituyen bases de datos híbridas que, a su vez, conforman constelaciones. Ejemplo de todo ello es el proyecto POSDATA.
\end{abstract}

KeYwords: Digital Humanities, Medieval Poetry, PALABRAS-ClAVE: humanidades digitales, poesía Textuality, Diffusion, Constellation medieval, textualidad, difusión, constelación

APROXIMACIONES AL ESTUDIO DE LA POESÍA MEDIEVAL EN EL SIGLO XXI: LAS HUMANIDADES DigitALES

Cuando hablamos de nuevas formas y espacios de circulación de la producción cultural en la actualidad estamos hablando, si bien no solo, principalmente de su presencia en el medio digital. En el campo de la investigación académica, como bien sostiene Klinenberg (2005: 6-18), el impacto de las tecnologías digitales puede medirse tanto desde aspectos prácticos como teóricos; tanto en lo que hace a los modos de producción y circulación como en lo que afectan a la colaboración y la autoría. No resulta extraño, entonces, que el formato, la normalización o la estandarización sean hoy, consecuentemente, parte de la agenda de investigación científica en el mundo. No obstante, en este proceso de tecnologización, la urgencia y la inmediatez lo nortean todo desde ese lugar tan difícil de definir: Internet. En palabras de Castells (2001: 2): «The 
Internet is a communication medium that allows for the first time, the communication of many to many, in chosen time, on a global scale ${ }^{1} \gg$.

En este trabajo queremos acercarnos al fenómeno de la necesidad de fijación y la circulación de la poesía — con especial énfasis en la medieval- en un medio como el digital, en el que la ubicuidad de la información lucha con el cambio constante y la evanescencia. Este tipo de manifestación cultural sufre un largo proceso de fijación que va, en muchos casos, de la transmisión oral a la fijación por parte de la imprenta y, más tarde, a su transformación y difusión como objeto digital (Kallinikos et al., 2010). Dejamos a un lado las manifestaciones que la llevan al ámbito de la divulgación para centrarnos, específicamente, en el campo de la investigación científica, principalmente ligado con las emergentes humanidades digitales (González-Blanco, 2014: 53-67; Rio Riande, 2015: 7-19; 2016a: 95-108; 2016b; 2016c).

Las Humanidades Digitales, como afirma Rio Riande (2016d), son y construyen objetos frontera (en términos de Star \& Griesemer, 1989, boundary objects), objetos de diversa naturaleza, comunes a varias disciplinas o comunidades, objetos que adquieren diferentes significados dependiendo del espacio en el que se insertan, pero que mantienen una estructura común que los hace reconocibles por esas comunidades: repositorios textuales digitalizados, ediciones digitales académicas colectivas o de autor, repertorios métricos, bases de datos, vocabularios controlados, etc. En el caso de nuestro trabajo, el corpus medieval se configura en estos objetos digitales a través de distintos tipos de textos (una edición pre-existente, una nueva edición digital, un conjunto de recursos, herramientas, etc.), entablando un diálogo contextual en el que reelaboración y reúso conviven con soluciones born digital ${ }^{2}$.

Un objetivo relacionado con la construcción de objetos frontera es el que desde hace tiempo nos propusimos en el grupo PoeMetCa, Recursos digitales para el estudio de la Poesía Medieval Castellana (González-Blanco et al., 2016a; Rio Riande: 2016e), y que terminó de cristalizarse en el proyecto de investigación POSTDATA, Poetry Standardization and Linked Open Data ${ }^{3}$. POSTDATA busca comparar las distintas tradiciones poéticas europeas — principalmente las medievales - a partir del análisis y estudio de objetos digitales de la más diversa naturaleza, utilizando las tecnologías de la web semántica. Desde esta aproximación hace dialogar a objetos digitales (sus objetos de estudio) y los resignifica desde la interoperabilidad semántica (González-Blanco et al., 2016b), para dar cuenta de los modos en los que la tecnología nos permite detectar patrones métricos, rimáticos y textuales, y arrojar luz sobre las formas de la poesía medieval en un marco pan-europeo.

\section{DIFUSIÓN ORAL, IMPRESA Y DIGITAL. EL PARÉNTESIS DE GUTENBERG}

Desde la aparición del seminal libro de Walter Ong, Orality and Literacy. The Technologizing of the Word, en $1982^{4}$, se ha venido señalando la irrupción de la imprenta en el siglo XV como factor de cambio en los modos de producción, distribución, difusión y consumo de los contenidos culturales en Occidente. Es, según el jesuita norteamericano,

\footnotetext{
${ }^{1}$ «La Internet es un medio de comunicación que permite por primera vez la comunicación de muchos a muchos, en un solo momento, a escala global» (trad. de las autoras).

${ }^{2}$ Una aproximación desde el ámbito de los repertorios métricos en papel a sus manifestaciones en formato digital es la que abordamos en González-Blanco et al. (2014a: 172-185; 2014b: 209-219).

3 <http://postdata.linhd.es/> (último acceso el 8 de marzo de 2017).

${ }^{4}$ De todos modos, Ramus, Method, and the Decay of Dialogue: From the Art of Discourse to the Art of Reason (1958) e Interfaces of the Word (1977), prefiguran y terminan de completar la idea de tecnologización de la palabra desarrollada en su Orality...
} 
apenas en el siglo XX cuando los avances tecnológicos permiten la recuperación de una parte de esa primera fase relacionada con la oralidad, si bien es una oralidad mediada por la escritura. Es este sentido en el que define Ong su bien conocida (y no exenta de polémica) tesis de la oralidad secundaria ${ }^{5}$ :

Al mismo tiempo, con el teléfono, la radio, la televisión y varias clases de cintas sonoras, la tecnología electrónica nos ha conducido a la era de la «oralidad secundaria». Esta nueva oralidad posee asombrosas similitudes con la antigua en cuanto a su mística de la participación, su insistencia en un sentido comunitario, su concentración en el momento presente, e incluso su empleo de fórmulas (Ong, 1996: 134).

Esta teoría parte, claro está, de la de Marshall McLuhan y su famoso libro The Gutenberg Galaxy (La Galaxia Gutenberg) de 1962.

De este punto de partida nace la idea del paréntesis de Gutenberg, formulada primeramente por el profesor Lars Ole Sauerberg (2009: 79-80). Sauerberg y Thomas Pettitt forman parte de un grupo de investigación del Institute for Literature, Media and Cultural Studies de la University of Southern Denmark que se ha centrado en desarrollar un proyecto llamado The Gutenberg parenthesis-print, book and cognition. The Gutenberg Parenthesis Research Forum ${ }^{6}$. Se trata de un foro abierto de discusión, que organiza eventos más o menos formales partiendo de la siguiente pregunta: «Is our emerging digital culture partly a return to practices and ways of thinking that were central to human societies before the advent of the printing press? ? $^{7}$.

En líneas generales, según las investigaciones de Sauerberg, la era de la textualidad sería, esencialmente, una interrupción, un breve paréntesis en la forma de comunicación y de creación y transmisión de la cultura, en la historia de la humanidad. $\mathrm{Su}$ teoría tiene, además, otro punto fuerte y es la concepción de la era digital, no solo como una fase distinta en una progresión lineal de las formas de comunicación, sino más bien como una vuelta a las prácticas y los modos de pensar anteriores a la imprenta. Sauerberg y Pettitt distinguen tres épocas con distintas características a la hora de producir un artefacto cultural y de la circulación-evolución de dicho artefacto. Así lo indica este gráfico de Pettitt (2007):

\footnotetext{
${ }^{5}$ Largo ha sido el debate sobre la oralidad primaria y secundaria. Para saber más, además de Ong (1982), se puede acudir, entre otros, a Havelock (1996), Cole (1991) o Quasthoff (1995).

${ }^{6}$ Después de varios cambios institucionales (fue parte del MIT), el proyecto está alojado actualmente en el sitio de la University of Southern Denmark en la dirección: <http://www.sdu.dk/en/om_sdu/institutter_centre/ikv/forskning/forskningsprojekter/gutenberg_projekt-> (último acceso el 7 de marzo de 2017).

7 «¿Es nuestra emergente cultura digital, en parte, un retorno a las prácticas y modos de pensar que eran centrales para las sociedades humanas antes del advenimiento de la imprenta?» (trad. de las autoras). La documentación del proyecto de Sauerberg, y los trabajos con Pettitt, que más adelante también retomamos, se encuentran hoy alojados en:

<http://www.sdu.dk/en/Om_SDU/Institutter_centre/Ikv/Forskning/Forskningsprojekter/Gutenberg_projek t/PositionPaper> (último acceso el 7 de marzo de 2017).
} 


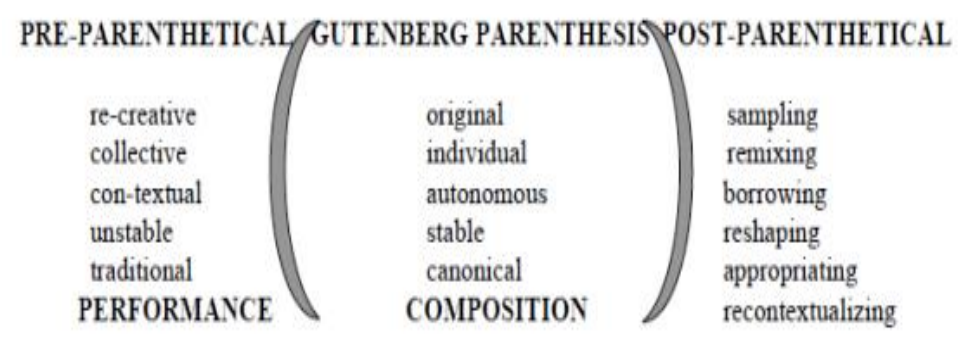

[Fig 1. Gráfico del paréntesis de Gutenberg y sus características (Pettitt, 2007)]

Desde esta reflexión, la era pre-gutenberg se caracterizaba por su fluidez. La cultura no se transmitía de manera escrita, por lo que era efímera, difícil de almacenar en un soporte físico, pero fácilmente compartible y flexible, es decir, podía ser modificada. Es la imprenta la que introduce una idea de fijación, de permanencia, de estabilidad y autoridad. La actual era digital hace que nos movamos a un nuevo paradigma, a uno mucho más cercano al anterior a la imprenta. En esta etapa se pierden la idea de autoridad, individualidad, fijación de la imprenta, y volvemos a una mayor fluidez, en la que la conexión hace que los objetos digitales sean considerados más colectivos, más propicios para poder ser apropiados por cualquiera y modificados, más aptos para usarse en otros contextos. En una entrevista a Pettitt y Sauerberg, el primero explica cómo la sensación de lo efímero contamina también el medio digital:

In my experience and my thinking, the Internet, digital technology are as ephemeral as speech in the sense that I cannot easily now access documents I wrote in 2003 (Pettitt y Sauerberg, 2013) ${ }^{8}$.

El cambio fundamental al que apuntan los autores es la concepción de la realidad más ligada a la idea de una red de conexiones que a una estructura de pertenencias. En este sentido, la cultura se transmitía de persona en persona en el periodo pre-imprenta, llegaba de alguien, cambiaba, se difundía. Con el libro pasa a estar contenida en un objeto. Pettitt defiende que Internet funcionaría más como esa red que como un contenedor:

DS: And so you're rejecting the idea of the Internet as a container?

Pettitt: Yes, I do. It's a network.

DS: And the computer file is not a container?

Pettitt: Yes it is, if it's static, and you can't manipulate it. You can even manipulate PDF now, can't you? A static computer file belongs within the Gutenberg Parenthesis because it can't be interfered with - it is contained; demarcated. That is the last form of the book, I think (Pettitt y Sauerberg, 2013) 9 .

\footnotetext{
8 «En mi experiencia y desde mi punto de vista, Internet, la tecnología digital, es tan efímera como el habla, en el sentido de que no puedo acceder fácilmente a los documentos que escribí en 2003» (Pettitt y Sauerberg, 2013) (trad. de las autoras).

${ }^{9}$ DS: ¿Y por eso rechaza la idea de Internet como contenedor?

Pettitt: Sí, lo hago. Es una red.

DS: ¿Y el archivo de computadora no es un contenedor?

Pettitt: Sí lo es, si es estático y no se puede manipular. Incluso puede manipularse un PDF ahora, ¿no? Un archivo de computadora estático pertenece al paréntesis de Gutenberg porque no se puede interferir lo que en él está contenido, demarcado. Creo que esa es la última forma del libro (Pettitt y Sauerberg, 2013) (trad. de las autoras).
} 
A pesar de ello, como Joaquín Rodríguez (2008; 2010), explican muy bien que el fenómeno de la imprenta es prefigurado por la historia de la escritura en general y que dicho paréntesis es:

[...] simplemente insostenible: Gutenberg no hizo sino instrumentalizar y seriar, reproduciéndola maquinalmente, la arquitectura de un artefacto que lo antecedía en más de once siglos. El códice precedió al libro en más de mil años, su estructura actual y la mayoría de sus dispositivos textuales estaba ya presente entonces. La historia concreta, por tanto, de eso que llamamos libro, de esa mediación específica al conocimiento, tiene unos 1700 años. El paréntesis, por tanto, comienza a ensancharse (Rodríguez, 2010).

Estas reflexiones, creemos, nos proporcionan un marco teórico muy sugerente para abordar la investigación de la lírica de la Edad Media en el siglo XXI. Siguiendo a todos estos autores podríamos, además, llegar a un lugar diferente para pensar el modo en el que el medio digital produce un cambio significativo sobre la textualidad, a mitad de camino entre la cultura pre-parentética y la parentética. Si bien es cierto que el medio digital actualiza procesos de circulación discursiva caracterizados por la inmediatez, la evanescencia, la transcripción actualizada por cada mediador y la legitimidad colectiva como modelo hegemónico, este tiene un cambio fundamental con respecto a la oralidad primaria y es la supremacía del algoritmo por sobre el contenido, la gramática por sobre la narración. Eso es un triunfo de la escritura, solo que ahora no se muestra a los ojos del lector, sino que permanece oculta en la estructura de una base de datos, de un programa. Así, ni siquiera la escritura (manuscrita o impresa) es tan dependiente de la estructura como lo es el texto digital, ya que toda la dinámica digital depende de la arquitectura algorítmica que la vehiculiza, la contiene y hace viable los cambios e intercambios en la web. Si la pre-parentética oralidad depende de la fonación y el oído, la post-parentética digitalidad descansa en el algoritmo y el modelo de datos. Su aparente desorden es tan estructurado como el de la letra impresa, solo que, en apariencia, en su front-end, tiene la evanescencia de la oralidad, y en su back-end la constricción de la escritura ${ }^{10}$.

\section{LA POESÍA MEDIEVAL EN EL MEDIO DIGITAL}

¿Cómo se transmite y circula en Internet la poesía? Más allá de los espacios divulgativos, donde la poesía aparece enmarcada en una actividad de lectura placentera, como en blogs o webs temáticas, u otras aproximaciones como la de la e-Poetry ${ }^{11}$, la poesía tiene, desde los inicios de la informática humanística (Humanities Computing), un lugar de privilegio en la investigación académica. Como bien explica Rommel (2004):

Right from the very beginning, humanities computing has always maintained its multidimensional character as far as literary genre, socio-cultural context and historic-

${ }^{10}$ Agradecemos las reflexiones de Diego Ferreyra sobre este tema, vertidas en el Workshop «Building a common model for semantic interoperability in the digital poetry ecosystems», organizado por el Laboratorio de Innovación en Humanidades Digitales (LINHD) como parte de la ERC Week en la UNED de Madrid, del 15 al 17 de marzo de 2017. Sobre las cuestiones relacionadas con la pragmática de los textos digitales, véase Fiormonte (2008: 65-84; 2010).

${ }^{11}$ Un ejemplo de esta otra aproximación a la poesía en formato digital, desde la idea del formato (subrayando su condición electrónica en el prefijo $e$ que la antecede) en: <http://iloveepoetry.com/> (último acceso el 7 de marzo de 2017). 
geographical provenance of literary texts is concerned. Studies have focused on poetry, drama, and narrative from antiquity to the present day ${ }^{12}$.

Es de destacar que pocos años pasan entre la aparición del primer sitio web en 1991, por el creador de la World Wide Web, Tim Berners-Lee, y, por ejemplo, la edición en línea de textos y paratextos de la Divina Comedia de Dante en el proyecto de Deborah Parker, The World of Dante ${ }^{13}$ en 1996 para el Institute for Advanced Technology in the Humanities de la Universidad de Virginia. De este modo, si bien la web nos sitúa en este espacio de dispersión y disolución de la autoridad del que hablaba Pettitt (2007), es cierto que la mayor parte de los proyectos que digitalizan, editan y/o trabajan en la explotación y difusión de este tipo de objetos digitales se enmarcan en instituciones académicas o a través de proyectos de investigación, con lo que, sobre este principio de disolución de la autoridad de la web, se impone otro que es de la institucionalidad.

¿Y qué es lo que encontramos en esta constelación? ${ }^{14}$ Objetos digitales, objetosfrontera, de la más diversa naturaleza: índices, catálogos, repertorios, bibliotecas digitalizadas, ediciones digitales acompañadas (o no) de imágenes, en interfaces que nos acercan versiones distintas (ediciones paleográficas, críticas, etc.), bases de datos híbridas, documentos enriquecidos con software de geolocalización, etc. Solo por poner unos pocos ejemplos que buscan dar cuenta de la naturaleza diversa de los objetos digitales, dentro del espacio de los proyectos de investigación dedicados al estudio de la poesía en los últimos treinta años podríamos mencionar al titánico Philobiblon, «base de datos bio-bibliográfica sobre textos romances escritos en la península ibérica en la Edad Media $^{15}{ }^{1}$, Cantus Database, una base de datos que contiene índices e imágenes de antifonarios, pero también grabaciones ${ }^{16}$, y que alimenta el ámbito de la lírica latina en formato digital conformada también por otros proyectos como el Corpus Rhythmorum Musicum $^{17}$, los Annalecta Hymnica Digitalia ${ }^{18}$ o Pedecerto ${ }^{19}$. En lo que respecta a la poesía profana, podríamos nombrar al Nouveau Naetebus ${ }^{20}$ dedicado a la lírica francesa narrativa medieval, o a las Cantigas de Santa María Database ${ }^{21}$ de la Universidad de Oxford, en lo que hace a la lírica mariana alfonsí; ambos, nodos bibliográficos y métricos. Otro conjunto relevante estaría compuesto por una serie de repertorios métricos digitales como la Bibliografia Elettronica dei Trovatori (BedT) ${ }^{22}$ o el Répertoire de la Poésie

12 «Desde sus inicios, la informática humanística mantuvo su carácter multidimensional en lo que hace al género, contexto sociocultural y la procedencia histórico-geográfica de los textos literarios. Sus estudios se han centrado en la poesía, el drama y la narrativa, desde la Antigüedad hasta nuestros días» (trad. de las autoras).

13 <http://www.worldofdante.org/> (último acceso el 8 de marzo de 2017).

14 Tomamos prestado el concepto de constelación, acuñado por el filósofo y crítico literario marxista Walter Benjamin (1842-1940), con el fin de acercarse a la historia y otras ciencias. Benjamin parte de la interpretación de las constelaciones de las estrellas, dado que estas, autónomas, independientes no tienen ninguna relación de dependencia mutua, pero nuestra observación nos ha llevado a establecer líneas de unión que se resumen en constelaciones. Véase Buck-Morss (2001).

15 <http://bancroft.berkeley.edu/philobiblon/index_es.html> (último acceso el 8 de marzo de 2017).

16 <http://cantusdatabase.org/> (último acceso el 8 de marzo de 2017).

$17<$ http://www.corimu.unisi.it> (último acceso el 8 de marzo de 2017).

$18<\mathrm{http}: / /$ webserver.erwin-rauner.de/crophius/Analecta_conspectus.htm (último acceso el 8 de marzo de 2017).

19 <http://www.pedecerto.eu> (último acceso el 8 de marzo de 2017).

$20<$ http://nouveaunaetebus.elte.hu> (último acceso el 8 de marzo de 2017).

$21<$ http://csm.mml.ox.ac.uk/> (último acceso el 8 de marzo de 2017).

$22<$ http://www.bedt.it/BEdT_04_25/inf_home_crediti.aspx> (último acceso el 8 de marzo de 2017). 
Hongroise ancienne $(R P H A)^{23}$. Bases de datos textuales y métricas serían, por ejemplo, la Base de Datos da Lírica Profana Galego-Portuguesa (MedDB2) ${ }^{24}$, para la lírica profana gallego-portuguesa medieval, The Last Song of the Troubadours ${ }^{25}$, para la poesía occitana tardía, Lyrik des deutschen Mittelalters ${ }^{26}$, para la poesía alemana medieval, o el Repertorio Métrico Digital de la Poesía Medieval Castellana (ReMetCa) ${ }^{27}$, para la compuesta en lengua castellana.

En esta constelación, la fijación del sentido está siempre en tensión, ya que el contenido es siempre mutable y todos estos objetos pueden dar cuenta de distintas versiones $^{28}$. No obstante, la aproximación académica, entendida desde un proyecto institucional que busca preservarse y ofrecerse, a la vez como voz autorizada, naturaliza la inestabilidad que suponen la dispersión, el carácter colectivo o la reutilización de recursos ${ }^{29}$ y los transforma en parte del proceso de investigación científica cada vez que recurrimos, como investigadores o usuarios, a estos objetos digitales como fuentes primarias para nuestros trabajos.

\section{POSTDATA. ORDEN EN EL CAOS}

¿Qué pasaría si esta constelación de objetos digitales, en la red que aquí demarcamos, pudiesen enriquecerse y ofrecer la mayor cantidad de información posible, y además compartiesen sus datos? A esto apunta lo que las ciencias de la información denominan interoperabilidad:

La posibilidad de que distintos tipos de ordenadores, redes, sistemas operativos, y aplicaciones trabajen juntos de forma eficaz, sin comunicación previa, de tal forma que puedan intercambiar información de manera útil y con sentido (Dublin Core Metadata Initiative, 2005).

Se trata de una idea muy sugerente y en consonancia con la de constelación, pues permite que los sistemas se comuniquen «mediante una interconexión libre, automática y transparente, sin dejar de utilizar en ningún momento la interfaz del sistema propio» (Gómez, 2007: 28).

Basado en las ideas de reutilización de recursos, de los datos de la literatura como objeto de investigación científica y de interoperabilidad, nace el mencionado proyecto de investigación POSTDATA (Poetry Standardization and Linked Open Data). Si bien no pretendemos aquí realizar una aproximación técnica, queremos señalar que POSTDATA se ha propuesto ordenar el universo post-parentético de la digitalidad con el fin de que la constelación de objetos digitales que estudia pueda ser interoperable, ofreciendo, para ello, un modelo de datos que pueda convertirse en un estándar para la comunidad académica interesada en el estudio de la poesía, en especial de la medieval.

El orden que propone POSTDATA, a partir de su análisis comparativo y su voluntad de estandarización, deja a la luz dos cuestiones de interés para el estudio de la poesía medieval europea. Desde lo filológico, como es de esperar, no hay terminología

\footnotetext{
$23<$ http://rpha.elte.hu/> (último acceso el 8 de marzo de 2017).

$24<$ http://www.cirp.es/bdo/med/meddb.html> (último acceso el 8 de marzo de 2017.

$25<$ http://icalia.es/troubadours/ca/> (último acceso el 8 de marzo de 2017.

26 <http://www.lhm-online.de> (último acceso el 8 de marzo de 2017.

$27<$ http://www.remetca.uned.es> (último acceso el 8 de marzo de 2017.

${ }^{28}$ Piénsese en el caso de la mencionada MedDB, que aquí se cita en su segunda versión, MedDB2.

${ }^{29} \mathrm{En}$ muchos casos, estos objetos digitales aprovechan textos que antes circularon en formato impreso (González-Blanco et al., 2014: 172-185).
} 
estándar que posibilite el diálogo entre los diversos objetos digitales que estudia, ya que las distintas tradiciones poéticas han apelado, a lo largo del tiempo, a distintas escuelas y teoría para explicarse. Consecuentemente, la mayor parte de los proyectos de investigación que se inscriben en estos objetos digitales siguen de cerca una u otra teoría poética a través de la terminología filológica que utiliza para expresarse. Desde el punto de vista informático tampoco hay normalización, ya que estos objetos digitales están basados en tecnologías distintas, muchas veces pertenecientes a épocas diferentes (desde bases de datos en DOS o Access a sistemas de etiquetado de textos con XML-TEI).

Este abordaje tecnológico no soslaya ni invalida su fuerte impronta filológica, ya que el estándar que propone desde lo tecnológico descansa en cimientos dedicados al estudio de la evolución de la métrica y versificación y de la bibliografía existente sobre este campo $^{30}$, para conocer el desarrollo de los términos y los conceptos. Una vez delimitados, los elementos conceptuales se abordan desde los objetos digitales mediante un proceso de ingeniería inversa (Curado Malta et al., 2017: 146-180). Cada uno de estos objetos digitales se estudia reconstruyendo su modelo de datos y pensando en cómo podría mapearse con el modelo estándar de POSTDATA que descansa, además, en una ontología para la poesía europea.

POSTDATA pone, de este modo, en práctica esa tensión entre front-end y backend, entre lo visible y lo invisible (Rio Riande 2016a: 95-108), a la que nos referíamos más arriba y deja a la luz la existencia de una web académica que busca fundarse en los principios del orden de la web semántica y sus tecnologías. Entonces, si bien es cierto que el medio digital acentúa la inmediatez, la evanescencia y la legitimidad colectiva como modelo hegemónico, lo que hace POSTDATA es ordenar a través de la preponderancia del algoritmo sobre el contenido, dando cuenta de cómo toda su dinámica depende de las arquitecturas, los lenguajes de marcado, los algoritmos y modelo de datos.

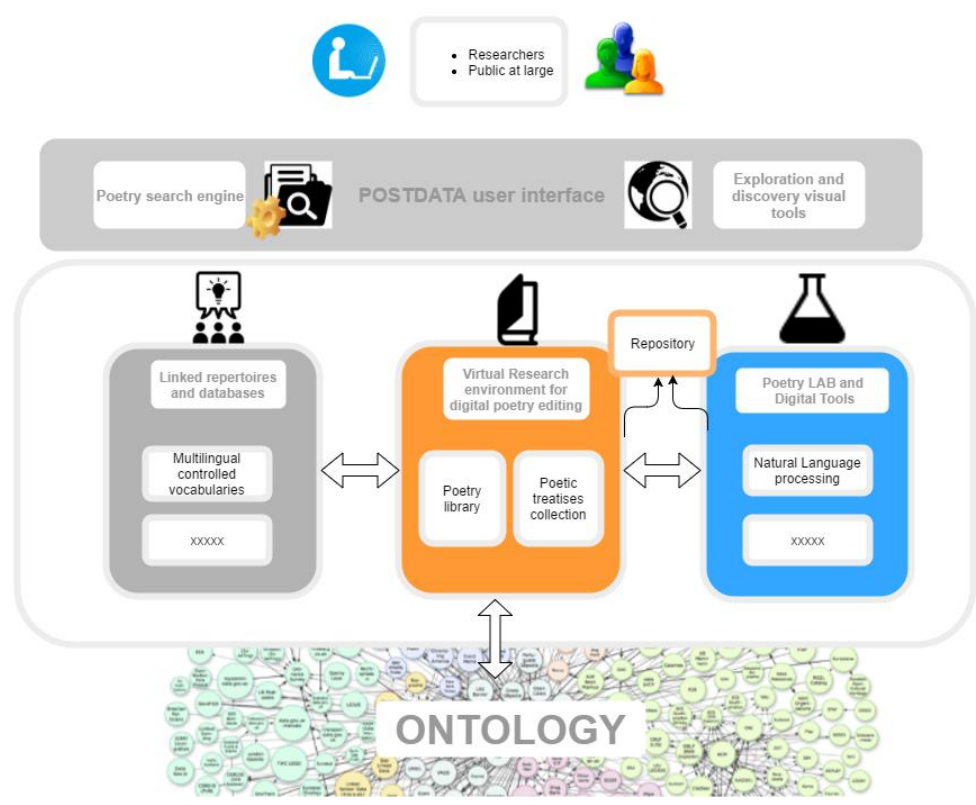

[Figura 2. El proyecto POSTDATA, <http://postdata.linhd.es/>]

${ }^{30}$ En español cabe destacar, por completo y reciente, la Historia de la métrica medieval castellana dirigida por Gómez Redondo (2016), punto de partida de todos los proyectos del grupo PoeMetCa y de POSTDATA. 


\section{AlgunAS CONCLUSIONES}

Las humanidades digitales empoderan la figura del filólogo dentro de un proceso tecnológico donde la naturaleza del texto ocupa un lugar central. Intentar explicar las tensiones que subyacen al medio digital con respecto de la investigación científica es tarea urgente. El trabajo que un proyecto académico como POSTDATA propone, creemos, da buena cuenta de teorizaciones que se han venido desarrollando hasta el momento con respecto a estos temas y las lleva a la práctica.

Siguiendo a Fiormonte (2003: 11), el mar de Internet es como el río de Heráclito: no podemos hundirnos en él dos veces. El medio digital acentúa la paradoja heracliteana acercando el texto a la idea de proceso. Si bien esto podría ser una verdadera amenaza para el desarrollo y el estudio de objetos digitales como actividad de investigación científica, lo profundo del río busca ser un lecho de gramática dispuesto a ordenar el caos, a ahogar la evanescencia de la digitalidad. 


\section{BIBLIOGRAFÍA}

BuCK-MORSS, Susan (1981): Origen de la dialéctica negativa: Theodor W. Adorno, Walter Benjamin y el Instituto de Frankfurt, México, Siglo XXI.

CASTELLS, Manuel (2001): The Internet Galaxy. Reflections on the Internet, Business, and Society, Oxford, Oxford University Press.

Cole, Thomas (1991): The Origins of Rhetoric in Ancient Greece, Baltimore, The Johns Hopkins University Press.

Curado Malta, Mariana, Centenera, Paloma y González-Blanco, Elena (2017): «Using Reverse Engineering to Define a Domain Model: The Case of the Development of a Metadata Application Profile for European Poetry», en Developing Metadata Application Profiles, M. Curado Malta, A. A. Baptista y P. Walk (coord), Hershey, IGI Global, pp. 146-180. DOI: https://doi.org/10.4018/978-1-5225-2221-8.ch007

Dublin CORE MetadATA INITIATIVE (2005) DCMI Glossary. URL: http://dublincore.org/documents/usageguide/glossary.shtml\#I

FIORMONTE, Domenico (2003): Scrittura e Filologia nella' era digitale, Torino, Bollati Boringhieri editore.

Fiormonte, Domenico (2008): «The Pragmatics of Digital Texts», en Atti del Convegno internazionale di studi, 8-10, M. Dardano, G. Frenguelli y E. De Roberto (eds), Roma, Aracne, pp. 65-84.

FIORMONTE, Domenico (2010): «Digital Encoding as a Hermeneutic and Semiotic Act: The Case of Valerio Magrelli», Digital Humanities Quarterly 4 (1).

URL: <http://www.digitalhumanities.org/dhq/vol/4/1/000082/000082.html>

GóMEZ, L. Felipe (2007): «Interoperabilidad en los Sistemas de Información Documental (SID): la información debe fluir», Códices: Revista de la Facultad de Ciencias Económicas y Sociales: Programa de Sistemas de Información y Documentación, 3 (1), pp. 23-39.

GómEZ REDONDO, Fernando (ed.) (2016): Historia de la métrica medieval castellana, Logroño, Cilengua.

GONZÁLEZ-BlanCo GARCíA, Elena (2013): «Actualidad de las Humanidades Digitales y un ejemplo de ensamblaje poético en la red: ReMetCa», Cuadernos Hispanoamericanos, 761, pp. 53-67.

GonzÁlez-Blanco García, Elena, Martínez Cantón, Clara, Martos PÉrez, María Dolores y Rio RiANDE, Gimena del (2014a): «Una propuesta de integración del sistema de formularios de bases de datos MYSQL con etiquetado TEI: ReMetCa, Repertorio digital de la métrica medieval castellana», en Humanidades Digitales: desafios, logros y perspectivas de futuro, S. López Poza, y N. Pena Sueiro (eds.), pp. 209-219.

URL: <http://www.janusdigital.es/anexos/contribucion.htm?id=19>

GonzÁlez-Blanco García, Elena, Martínez Cantón, Clara, Martos Pérez, María Dolores y RIO RIANDE, Gimena del (2014b): «La codificación informática del sistema poético medieval castellano, problemas y propuestas en la elaboración de un repertorio métrico digital: ReMetCa», en Visibilidad y divulgación de la investigación desde las Humanidades Digitales. Experiencias y proyectos, Á. 
Baraibar, (ed.), Pamplona, Servicio de Publicaciones de la Universidad de Navarra [Biblioteca Áurea Digital, BIADIG, 22], pp. 172-185.

URL: <http://www.unav.edu/publicacion/biblioteca-aurea-digital/BIADIG-22>

GonZÁlez-Blanco García, Elena, Martínez CANTón, Clara, Martos PÉrez, María Dolores y Rio RiANDE, Gimena del (2016a): «Poemetca qué es (y qué queremos que sea)».

URL: <http://dayofdh2016.linhd.es/poemetca/2016/04/05/poemetca-que-es/>

GonzÁlez-Blanco García, Elena, Martínez CANTón, Clara, Martos PÉrez, María Dolores y Rio RIANDE, Gimena del (2016b): «Linked open data to represent multilingual poetry collections. A proposal to solve interoperability issues between poetic repertoires», en Proceedings of the LREC 2016 Workshop «LDL 2016-5th Workshop on Linked Data in Linguistics: Managing, Building and Using Linked Language Resources», J. P. McCrae, Ch. Chiarcos et al. (eds.) Portoroz, European Language Resources Association.

URL: <http://www.lrec-conf.org/proceedings/lrec2016/workshops/LREC2016 Workshop-LDL2016_Proceedings.pdf >

GonZÁleZ-Blanco García, Elena y SPENCE, Paul (2014): «A historical perspective on the digital humanities in Spain».

URL: 〈https://networks.h-net.org/node/63265/pdf>

HAVELOCK, Eric A. (1996): La musa aprende a escribir: reflexiones sobre oralidad y escritura desde la Antigüedad hasta el presente, Barcelona, Paidós. URL: https://dialnet.unirioja.es/servlet/libro?codigo $=81424$

Kallinikos, Jannis; Aaltonen, Aleksi y Marton, Attila (2010): «A theory of digital objects», en First Monday. DOI: https://doi.org/10.5210/fm.v15i6.3033

URL: <http://firstmonday.org/ojs/index.php/fm/article/view/3033/2564>

KLINENBERG, Eric (2005): «Cultural Production in the Digital Age: An Introduction», Annals of the American Academy of Political and Social Science, 597, pp. 6-18. DOI: https://doi.org/10.1177/0002716204270420

Ong, Walter J. (1958): Ramus, Method, and the Decay of Dialogue: From the Art of Discourse to the Art of Reason, Cambridge MA, Harvard UP.

ONG, Walter J. (1996): Oralidad y escritura: Tecnologías de la palabra, México, Fondo de Cultura Económica.

ONG, Walter J. (1977): Interfaces of the Word, Ithaca, Cornell UP.

PETTitT, Thomas (2007): «Media Studies and Mediaeval Studies: Bracketing the Gutenberg Parenthesis», en Guest lecture at Research Seminar in Media and Communications Studies, Institute for Culture and Communication, Södertörn University College, Stockholm.

PetTitT, Thomas y SAUERBERG, Lars Ole (2013): The future is medieval. A discussion with the scholars behind the «Gutenberg Parenthesis» a sweeping theory of digital - and journalism - transformation.

URL: 〈http://www.cjr.org/the_audit/the_future_is_medieval.php>

QUASTHOFF, Uta. M. (ed.) (1995): Aspects of oral communication, XXI, Berlin, New York, Walter de Gruyter. DOI: https://doi.org/10.1515/9783110879032

Rio Riande, Gimena del (2015): «Humanidades Digitales. Mito, actualidad y condiciones de posibilidad en España y América Latina», ArtyHum, monográfico, 1, La realidad de las Humanidades Digitales en España y América Latina, pp. 719. URL: 
<https://www.artyhum.com/descargas/monograficos/MONOGR\%C3\%81FICO $\% 20 H D . p d f>$

RIO RIANDE, Gimena del (2016a): «De todo lo visible y lo invisible o volver a pensar la investigación en humanidades digitales», Signa: Revista de la Asociación Española de Semiótica, 25, pp. 95-108.

DOI: https://doi.org/10.5944/signa.vol25.2016.16943

URL: <http://revistas.uned.es/index.php/signa/article/view/16943>

Rio RiANDE, Gimena del (2016b): «Ada en la encrucijada de las Humanidades Digitales», Luthor, 29.

URL: <http://www.revistaluthor.com.ar/spip.php?article149>

Rio RiANDE, Gimena del (2016c): «Humanidades Digitales: estándares para su consolidación en el campo científico argentino», Bibliotecarios: integración, identidad regional y abordaje transversal.

URL: <http://www.abgra.org.ar/documentos/48RNB_20160419_1400-Ponencia. pdf $>$

Rio RiAnde, Gimena del (2016d): Humanidades Digitales: Construcciones locales en contextos globales. URL:

$<$ http://sedici.unlp.edu.ar/blog/2016/03/22/humanidades-digitalesconstrucciones-locales-en-contextos-globales/>

RIO RIANDE, Gimena del (2016e): «Después del paréntesis de Gutenberg. El estudio y la edición digital de poesía», conferencia dictada en el marco del curso de verano del LINHD: Tecnologías digitales aplicadas al estudio de la poesía, del 27 de junio al 1 de julio de 2016 en la UNED de Madrid (España).

URL: <http://linhd.es/p/dhsummer2016/>

RoDRÍGUEZ, Joaquín (2008): Edición 2.0 Sócrates en el hiperespacio, Barcelona, Melusina.

RODRÍGUEZ, Joaquín (2010): El paréntesis de Gutenberg.

URL: <http://www.madrimasd.org/blogs/futurosdellibro/2010/05/25/132011\#. WNboVvnyuM8>

Rommel, Thomas (2004): «Literary Studies», en A Companion to Digital Humanities, S. Schreibman, R. Siemens y J. Unsworth (eds.), Oxford, Blackwell.

URL: <http://www.digitalhumanities.org/companion/>

SAUERBERG, Lars Ole (2009): «The Gutenberg Parenthesis-Print, Book and Cognition», Orbis Litterarum, 64 (2), pp. 79-80. DOI: https://doi.org/10.1111/j.16000730.2009.00962.x

STAR, Susan Leigh y GRIESEMER, James R. (1989): «Institutional Ecology, 'Translations' and Boundary Objects: Amateurs and Professionals in Berkeley's Museum of Vertebrate Zoology, 1907-39», Social Studies of Science, 19, pp. 387-420. DOI: https://doi.org/10.1177/030631289019003001 